\title{
The Indian summer monsoon as revealed by the NCMRWF system
}

\author{
P L S Rao, U C Mohanty, P V S Raju and Gopal IyengaR* \\ Centre for Atmospheric Sciences, Indian Institute of Technology Delhi, Hauz Khas, New Delhi 110016 , India. \\ * National Centre for Medium Range Weather Forecasting, Mausam Bhavan Complex, Lodi Road, \\ New Delhi 110 003, India.
}

\begin{abstract}
In this study, we present the mean seasonal features of the Indian summer monsoon circulation in the National Centre for Medium Range Weather Forecasting (NCMRWF) global data assimilation and forecast system. The large-scale budgets of heat and moisture are examined in the analyzed and model atmosphere. The daily operational analyses and forecasts (day 1 through day 5) produced for the summer seasons comprising June, July and August of 1995 and 1993 have been considered for the purpose. The principal aim of the study is two-fold. Primarily, to comprehend the influence of the systematic errors over the Indian summer monsoon, secondarily, to analyze the performance of the model in capturing the interseasonal variability.

The heat and moisture balances show reduction in the influx of heat and moisture in the model forecasts compared to the analyzed atmosphere over the monsoon domain. Consequently, the diabatic heating also indicates reducing trend with increase in the forecast period. In effect, the strength of Indian summer monsoon, which essentially depends on these parameters, weakens considerably in the model forecasts. Despite producing feeble monsoon circulation, the model captures interseasonal variability realistically. Although, 1995 and 1993 are fairly normal monsoon seasons, the former received more rainfall compared to the latter in certain pockets of the monsoon domain. This is clearly indicated by the analyzed and model atmosphere in terms of energetics.
\end{abstract}

\section{Introduction}

The Indian summer monsoon is a spectacular manifestation of regional scale anomalies of the general circulation of the atmosphere. It is a thermally driven circulation whose existence is attributed primarily to land sea heating contrasts. The complexity of the summer monsoon is augmented by the close interaction between the large-scale flow and moist convection. Since tropical regions are predominantly driven by agrarian economies, the vagaries in the monsoon rainfall beget severe ramifications. It is also known that the agriculture production invariably depends on the rainfall as surplus rainfall results in abundant produce of crops and deficient rainfall in severe famines. In this vein, the comprehension of variability of the summer monsoon from one season to another is very crucial. The seasonal mean climate is characterized by horizontal and vertical gradients of wind, temperature and moisture that are favorable for the growth of thermodynamic and hydrodynamic instabilities. The day-to-day weather fluctuations are produced by transient weather disturbances, which are manifestations of the growth, decay and propagation of these instabilities. These disturbances can derive their energy from the mean circulation and thereby change the mean circulation itself. The observed mean climate is produced by interactions among the stationary and quasi-stationary forcing functions and transient disturbances. The interseasonal variability of the summer monsoon is associated with the variability of either internal dynamics or boundary parame-

Keywords. Summer monsoon; energy budgets; systematic errors; interseasonal variability. 
ters such as sea surface temperature, soil moisture and snow. The seasonal rainfall is considered to be an integrated view of the seasonal climate. Since rainfall is the resultant of various forcing mechanisms, its variations are manifested in energetics and dynamics. Hence, a comprehensive knowledge of the dynamics and energetics is indispensable to the understanding of the interseasonal variability of the summer monsoon.

Although a large number of studies have been carried out on large-scale atmospheric energetics, a few are related to the Indian summer monsoon (Rao and Rajamani 1972; Mohanty et al 1983; Pearce and Mohanty 1984) through the analysis of heat and moisture budgets. By and large, most of these are case studies and restricted to selective domains and certain terms of the large-scale budgets. Further, there are some studies (Kanamitsu and Krishnamurti 1978; Krishnamurti et al 1989, 1990; Ramesh et al 1996), which focused on a pair of extreme (surplus and deficient) years and expounded the variability using various largescale meteorological fields. Nevertheless, there is no study, which elucidates the differences between a pair of normal years. Although the difference between two normal monsoon years is small, yet we experience the variability. In this study, we have addressed how that variability can be diagnosed and captured by the model. We demonstrate how the rainfall variations are manifested in largescale energetics and dynamics in the analyzed and model atmosphere over the Indian summer monsoon domain. In turn, we also elucidate the performance of the National Centre for Medium Range Weather Forecasting (NCMRWF) model in simulating observed interseasonal variability through the comprehensive analysis of heat and moisture budgets.

\section{Methodology}

The budget equations are obtained from the equations of motion and other conservation laws, which the atmosphere obeys. It is well known that at any time mean atmospheric circulation can be bifurcated into a stable mean component, which varies slowly, and another rapidly varying eddy component, which varies swiftly over a time period. In general, tropical circulations are driven by mean component of the flow and extratropical circulations by eddy component of the flow. Since, Indian summer monsoon circulation is predominantly driven by mean component of the flow, we have bifurcated the time mean into the respective mean and eddy budgets. In this study, we have elucidated the mean budgets only. The various bud- get equations are expressed in the flux form with pressure as the vertical coordinate.

The sensible heat budget equation is written as

$$
\frac{\overline{\partial\left(C_{P} T\right)}}{\partial t}+\nabla \cdot\left(C_{P} \bar{T} \bar{V}\right)+\frac{\partial\left(C_{P} \overline{\omega T}\right)}{\partial P}-\bar{\omega} \bar{\alpha}=\bar{Q}_{H} .
$$

In equation (1), the first term on the left describes the local tendency of enthalpy. The second and third terms designate the horizontal and vertical divergence fluxes of heat respectively. The fourth term indicates the adiabatic conversion of available potential energy to kinetic energy. The term on the right describes the contributions from all diabatic processes in the atmosphere namely radiation, condensation, evaporation of falling raindrops and turbulent transfer of sensible heat.

The latent heat budget equation is denoted as

$$
\frac{\overline{\partial(L q)}}{\partial t}+\nabla \cdot L \overline{q V}+\frac{\partial}{\partial P}(L \bar{\omega} \bar{q})=\bar{Q}_{L} .
$$

In equation (2), the first term on the left describes the local variation of latent heat. The second and third terms represent the horizontal and vertical divergence fluxes of latent heat respectively. Similarly, the term on the right connotes the moisture source/sink, which comprises diabatic heating due to latent heat released from evaporation and condensation as well as turbulent transfer of latent heat.

In the budget equations, the terms representing local tendency over a period of 92 days have negligible contribution towards the respective budgets. The results at each regular latitude-longitude grid points of $1.5^{\circ}$ are averaged both in zonal and meridional directions over the summer monsoon region and integrated vertically from 1000 to $100 \mathrm{hPa}$ to construct geographical distributions, vertical profiles and volume integrated budgets.

The volume integration of the budget terms with the boundary conditions that $\omega$ vanishes at the bottom and top of the atmosphere leads to elimination of the terms representing vertical flux divergence of various quantities. The discussion in this paper is confined to those terms, which contribute significantly to the respective large-scale balances and also responsible for the maintenance of the summer monsoon circulation.

\section{Data and scheme of analysis}

The database employed in this study consists of uninitialized analyses (0000 UTC) and forecasts (day 1 through day 5) of NCMRWF, India for June, 
July and August of 1995 and 1993. The reason for choosing these particular years is as follows. Both of these years are fairly normal with slight variation in terms of rainfall. As explained earlier, we endeavored to examine how this variability is reflected in diagnostic fields. The basic meteorological fields considered for the study include wind, temperature and moisture over the summer monsoon region extending from $15^{\circ} \mathrm{S}$ to $45^{\circ} \mathrm{N}$ latitudes and $30^{\circ} \mathrm{E}$ to $120^{\circ} \mathrm{E}$ longitudes at ten pressure levels $(1000,850,700,500,400,300,250,200,150$, $100 \mathrm{hPa}$ ) at a horizontal resolution of $1.5^{\circ}$ latitude/longitude. The mass and velocity fields are analyzed by three-dimensional data assimilation scheme. In this scheme, the assimilation is carried out in two different steps, viz., spectral statistical interpolation, and six hour forecast which provides the first guess in order to carryout the subsequent analysis.

In order to overcome the model bias, in the representation of the vertical velocity obtained from the spectral statistical interpolation procedure, we have not made use of that field stored in the NCWRWF archives. Instead, we derived it employing kinematic technique (O'Brien 1970) using uninitialized wind fields in this study with a constraint that the vertical velocity vanishes $(\omega=$ $\mathrm{d} P / \mathrm{d} t)$ at the bottom and top of the atmosphere (i.e., $\omega=0$ at $P=1000$ and $100 \mathrm{hPa}$ ). The consistency of the vertical motion thus computed represents semi-permanent circulation (Hadley cell) in more realistic manner over the tropics (Rao et al 1998). The vertical velocity fields computed by employing the kinematic technique are utilized in this study to estimate the large-scale balances. The various space derivatives are evaluated by centered difference scheme. All the budget terms are computed at each regular latitude/longitude grid points and integrated suitably over the domain of the atmosphere to obtain vertical profiles, vertical integrated geographical distributions and volume integrated budget parameters.

\section{Results and discussion}

The detailed analysis of significant terms of largescale budgets of heat and moisture is presented to illustrate the characteristic features of the Indian summer monsoon circulation. Also, the performance of the model in capturing the observed interseasonal variability is evaluated through diagnostic aspects.

\subsection{Systematic errors}

A priori knowledge of systematic errors of the model is indispensable to interpret various forecasts. The presence of systematic errors in the numerical models is attributed to improper representation of various physical processes and interaction among large-scale and small-scale processes. Therefore, a comprehensive knowledge of physical processes which influence and are largely responsible for the maintenance of the summer monsoon and the necessary parameterizations to represent them suitably in the general circulation models are vital to predict the summer monsoon circulation better in various time scales. The physical processes are generally represented by the expressions of certain conceptual formulations of unresolved scales of motion in terms of resolvable scales of atmospheric circulation models. The dependence of systematic errors of the seasonal scale on the parameterization of physical processes was amply demonstrated (Tiedtke et al 1988, Slingo et al 1988). The systematic flow field errors of the NCMRWF model are examined in the studies of Mohanty et al (1995) and Rao et al (1998). The main error characteristics are summarized as follows. The lower tropospheric $(850 \mathrm{hPa})$ flow field denotes weakening of southeasterly trades and the cross-equatorial flow $\left(2-4 \mathrm{~ms}^{-1}\right)$ into the Northern Hemisphere. The upper tropospheric $(200 \mathrm{hPa})$ biases in the circulation features include weakening of Tibetan anti-cyclone, and reduction of return flow into the Southern Hemisphere. The lower tropospheric $(850 \mathrm{hPa})$ temperature errors are depicted in figure 1. Analysis of temperature forecasts reveals that the entire monsoon domain is dominated by cooling in the lower levels. However, warming is noticed over northwest India, and Arabia. The established temperature gradient also demonstrates that the monsoon westerlies weaken with height and this trend increases with increase in the forecast period. The moisture forecasts (figure 2) show a drying of the model atmosphere over the monsoon region dominating in the lower levels, with sectors of moderate moistening delineated particularly over the Arabian Sea and northeast India. The general characteristics of the temperature and moisture forecast errors are analyzed through their corresponding sectorial $\left(30^{\circ} \mathrm{E}-120^{\circ} \mathrm{E}\right)$ mean pressure-latitude cross sections. The temperature and moisture forecast errors are depicted in figures 3 and 4 respectively for the monsoon season of 1995. The model forecasts show cooling in the entire troposphere except middle and lower troposphere north of $20^{\circ} \mathrm{N}$, where predominant warming is noticed. It is apparent that temperature errors in the forecasts are reasonable $\left(1-2^{\circ} \mathrm{K}\right)$ up to day 5 . The general cooling of the middle and upper tropospheric layers is due to the deficiencies in the long wave radiation parameterization. Nevertheless, the lower troposphere cooling (900-700 hPa) demands considerable atten- 


\section{Temperature $850 \mathrm{hPa}$ JJA 1995}
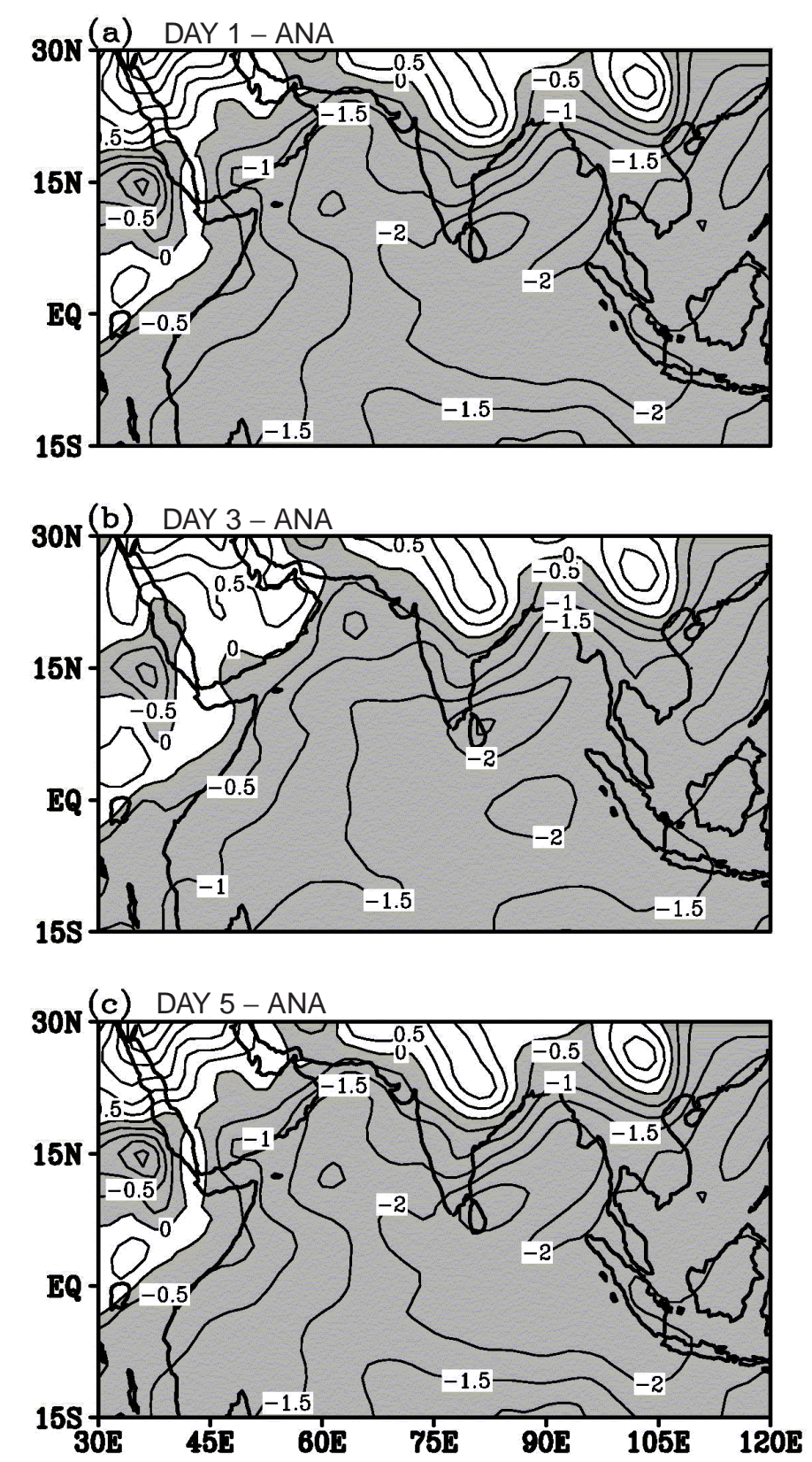

Figure 1. Geographical distribution of temperature forecast errors at $850 \mathrm{hPa}$ for JJA 1995 (units: ${ }^{\circ} \mathrm{K}$ ); (a) Day 1 error, (b) Day 3 error, (c) Day 5 error. (Contour interval: 0.5, negative values are shaded).

tion. This is ascribed to the treatment of shallow convection, which is expected to offset the radiational cooling. Incidentally, this is not effective in minimizing the radiational effects.

The sectorial mean moisture forecast errors show general drying of the model atmosphere in day 3 and day 5. Hence, the present analysis suggests, that the part of the moisture, which is available for moistening the environment has to be more in order to reduce the share of moisture, which heats up the atmosphere. Such a measure could lead to the general enhancement of precipitation in the model forecasts to offset the overall cooling of the model atmosphere. The overall effect of these errors is feeble monsoon circulation. Further, the zone of easterlies in the upper troposphere also gets shrunk over the monsoon region (Rao et al 1998). The subsequent discussion enunciates the influence of these errors on the summer monsoon circulation. 
Moisture $850 \mathrm{hPa}$ JJA 1995
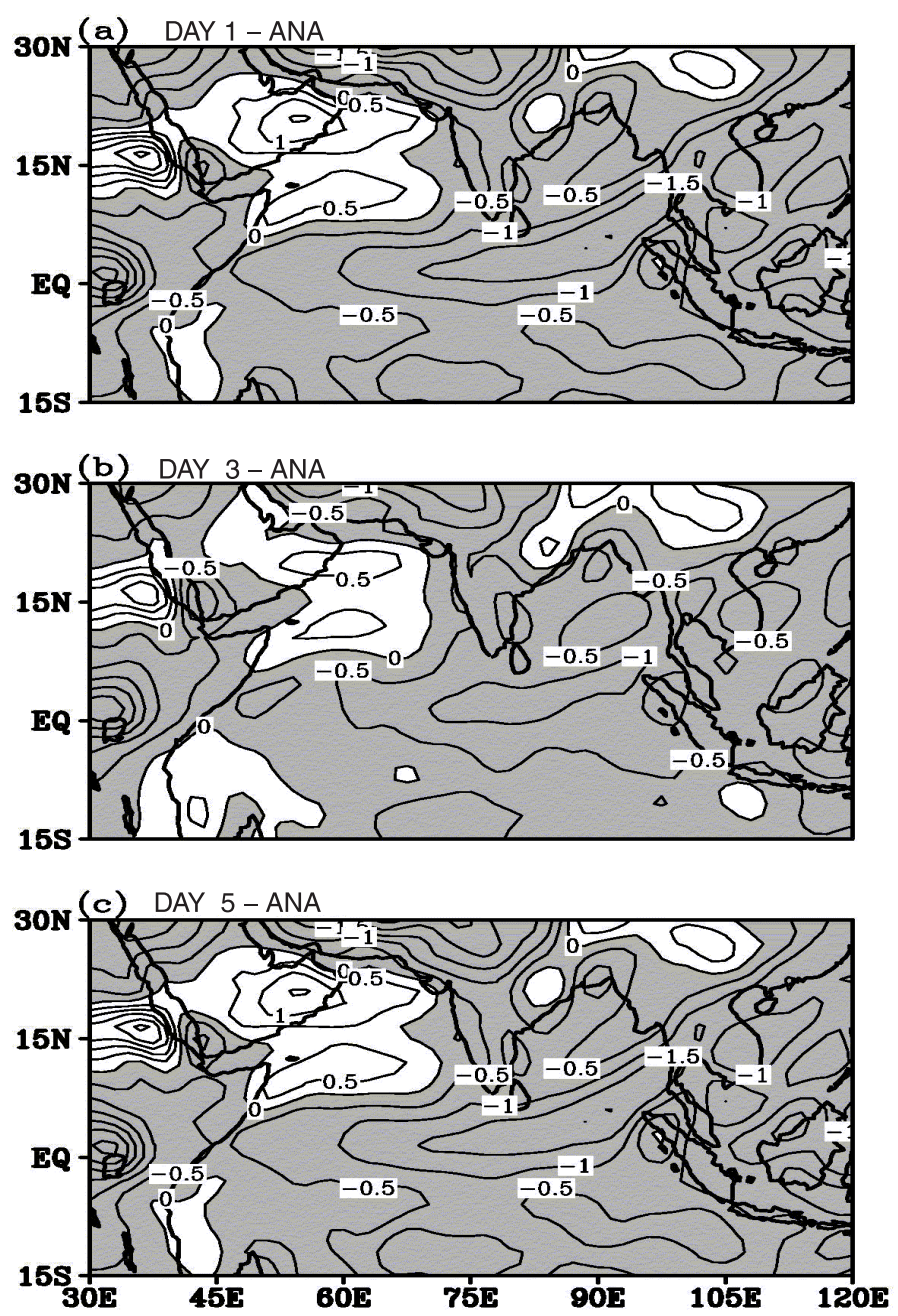

Figure 2. As in figure 1, but for moisture forecast errors (units: $\mathrm{gm} \mathrm{kg}^{-1}$ )

\subsection{Heat and moisture budgets}

We attempt to elucidate how the interseasonal variability of the Indian summer monsoon in terms of rainfall could be diagnosed using energetics. Our intension is manifold. Making use of this diagnostic analysis, one can ascertain whether a particular season is a surplus, normal or deficient monsoon season. Further, when we carry out the simulations of various scales of atmospheric systems, it is expected that they retain the mean features of these systems. Primarily, how the analyses reflect the observed rainfall variability in terms of mean diagnostic fields. Since rainfall is considered to be the resultant of various forcing mechanisms, whether these diagnostic fields capture the essential signature in the rainfall. Secondarily, how the model simulates the stable mean monsoon circulation. The performance of any numerical model is judged, based on how it represents the mean and transient eddy components of any atmospheric circulation. The thermodynamical characteristic features of the monsoon circulation represented by the analyses and forecasts of NCMRWF are analyzed through the heat and moisture budgets. There are some earlier studies (Savijarvi 1980, 1981; White 1988), which elucidate the budgets using ECMWF and NCEP model forecasts over the mid-latitudes. The present study augments similar efforts over the monsoon domain.

The vertical profile of the heat budget terms for the monsoon season of 1995 is depicted in figure 5. The horizontal flux divergence of heat (figure 5a) shows two maxima over the monsoon region. The convergence maximum is noticed in the lower troposphere and the divergence maximum in the upper troposphere. On the contrary, the vertical flux divergence of heat (figure 5b) indicates strong divergence in the lower troposphere and convergence in the upper troposphere. Thus, 
TEMPERATURE

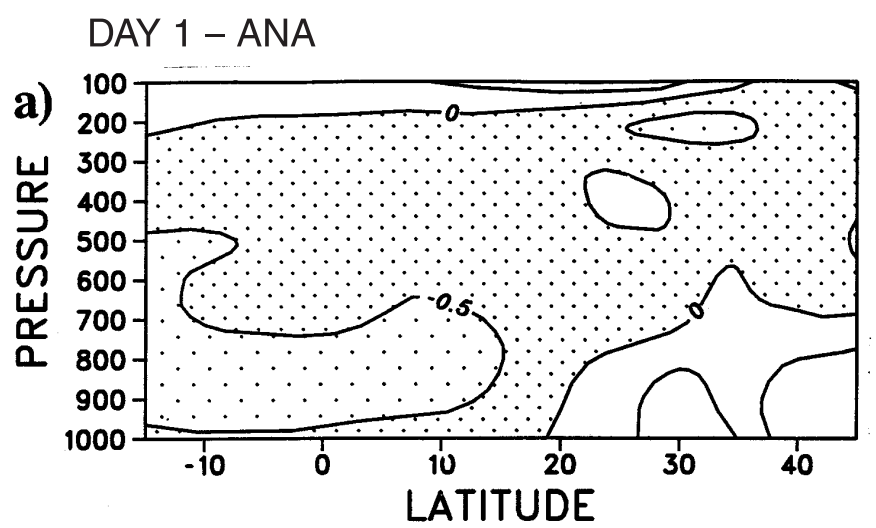

DAY 3 - ANA

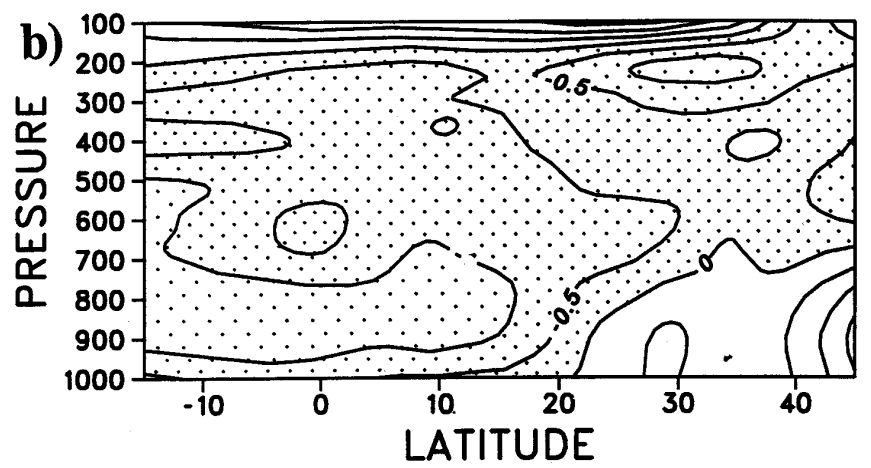

DAY 5 - ANA

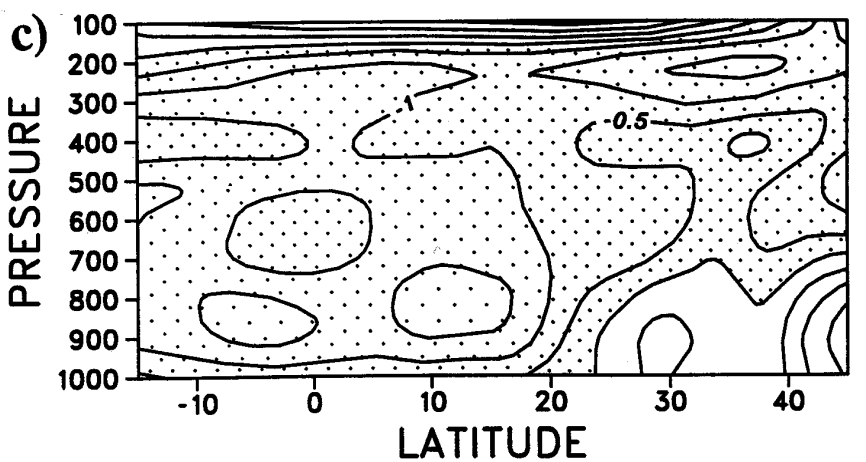

Figure 3. Sectorial $\left(30^{\circ} \mathrm{E}-120^{\circ} \mathrm{E}\right)$ mean pressure latitude cross sections of temperature forecast errors for JJA 1995 (units: $\left.{ }^{\circ} \mathrm{K}\right)$; (a) Day 1 error, (b) Day 3 error, (c) Day 5 error. (Contour interval: 0.5, negative values are shaded).

the horizontal flux of heat to a large extent balances the vertical flux in the heat budget. An earlier study (Mohanty et al 1983) reveals that the magnitudes of horizontal and vertical flux divergences of heat are very large and produce counter balance. In the summer monsoon domain, the horizontal flux divergence is dominant at upper tropospheric levels and vertical divergence flux in the lower tropospheric levels. The forecasts fairly simulate the trends of the horizontal and vertical divergence fluxes of heat. However, they underestimate these flux divergences in the lower as well as upper troposphere. The underestimation of these fluxes in the lower troposphere is attributed to two significant errors of the model, namely underestimation of the cross equatorial flow and general cooling over the monsoon region in the lower troposphere. Similarly, the weak Tibetan anti-cyclone, reduction in the return flow and cooling produced by the model are responsible for upper tropospheric underestimation. Since the systematic errors of the model delineate increasing trend with increase in the forecast period, the flux divergences also get enfeeble with the forecast period. The consistency of the characteristic features of the flux parameters in the heat budget is adduced by earlier 

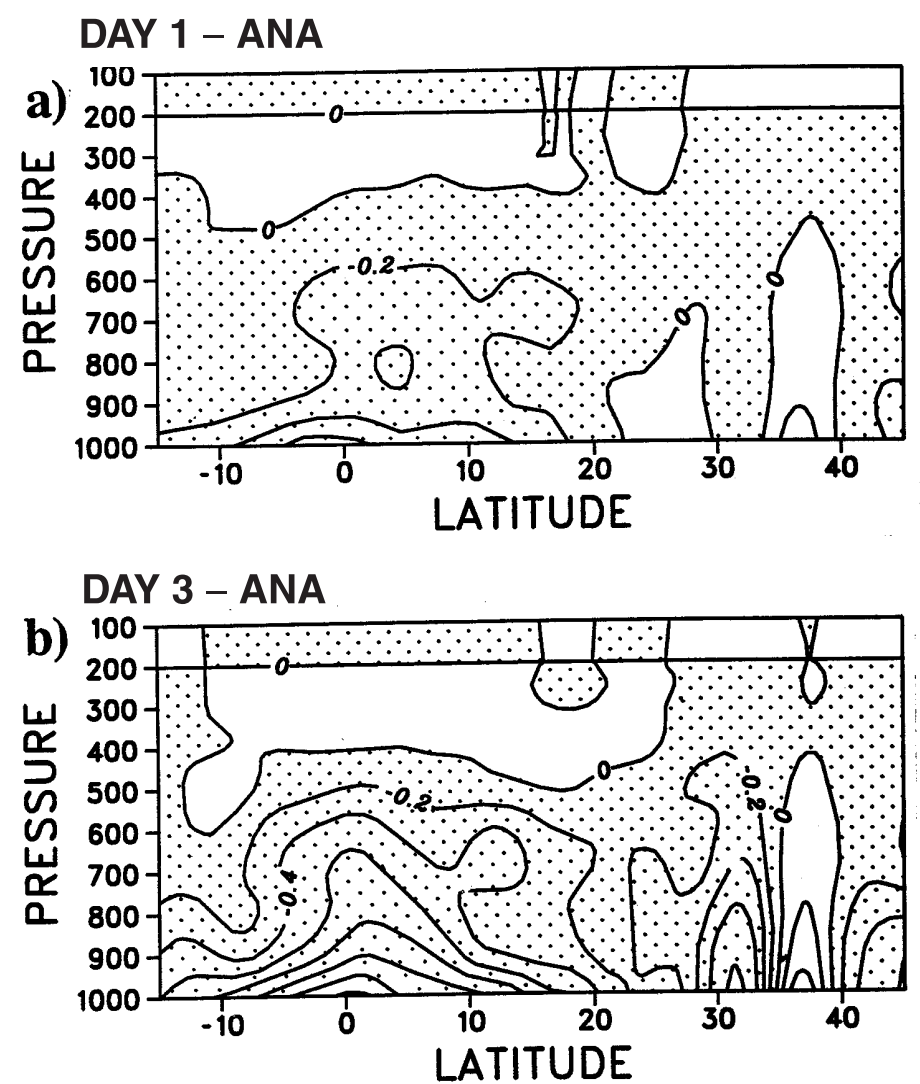

DAY 5 - ANA

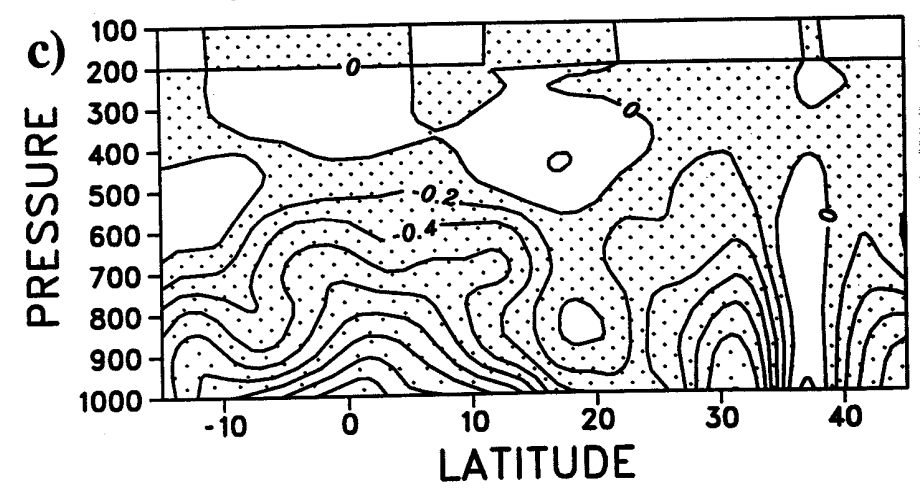

Figure 4. As in figure 3, but for moisture forecast errors. (Contour interval: 0.2, negative values are shaded).

studies (Savijarvi 1980, 1981 and Mohanty et al 1983).

The vertical variation of adiabatic conversion of kinetic energy from the available potential energy (figure 5c) shows maximum conversion around $300 \mathrm{hPa}$ level. This is attributed to the fact that the maximum amount of latent heat is released in the upper middle troposphere. The release of latent enhances the diabatic heating. Which in turn facilitates the increase in generation of available potential energy. As the increase in generation is supported by intense vertical rising motion the available potential energy (APE) available for conversion also increases. The maximum conversion depicted around $300 \mathrm{hPa}$ level is in good agreement with the earlier findings (Nitta 1970) over the tropics. Although the forecasts captured the conversion of APE to kinetic energy very well, they depict weak conversion with increase in the forecast period. In general, the forecasts in all ranges underestimate the conversion. Further, this trend increases with increase in the forecast period. The reduction in the flux convergence of heat begets reduction in the diabatic heating as a consequence 
HEAT BUDGET
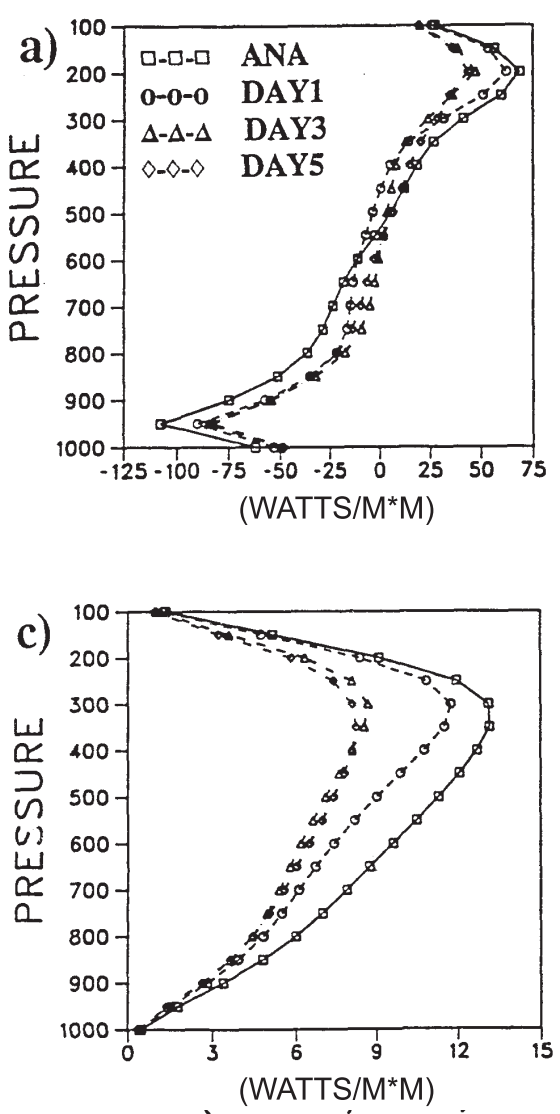

JJA 1995
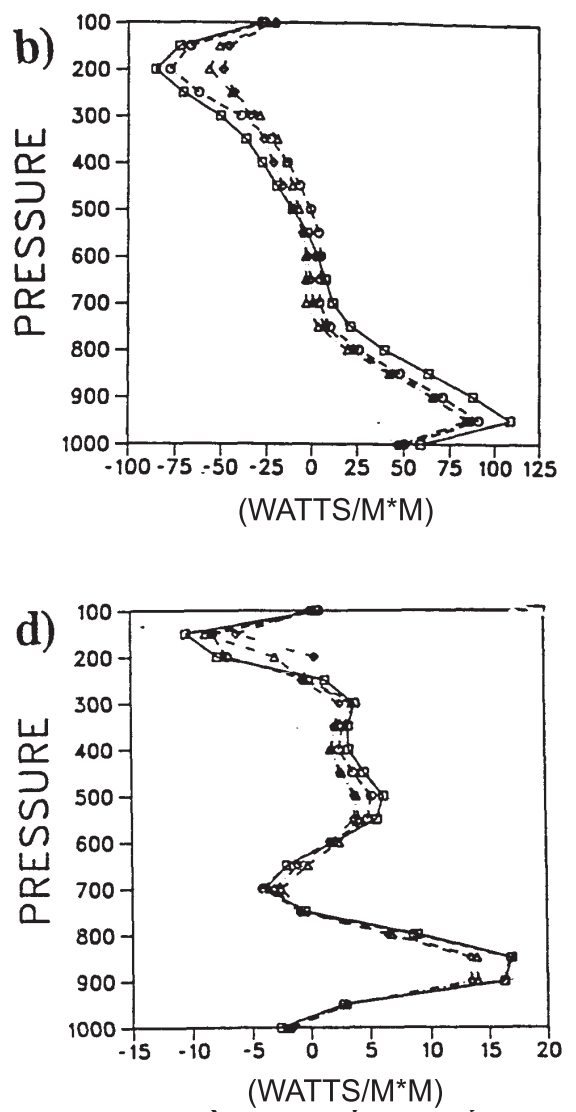

Figure 5. Area averaged vertical profile of heat budget terms for JJA 1995; (a) Horizontal flux divergence of heat, (b) vertical flux divergence of heat, (c) adiabatic conversion of available potential energy to kinetic energy, (d) diabatic heating.

of decrease in vertical motion followed by decrease in release of latent heat. The adiabatic generation confirms an important observation, the presence of a thermally driven circulation (direct cell) over the monsoon region. Profound vertical motions are characteristic features of the monsoon region, which give rise to generation of abundant available potential energy, which in turn gets transformed to kinetic energy. The prolific generation of kinetic energy enables the monsoon domain as a source region of kinetic energy.

The vertical profile of diabatic heating (figure 5d) shows heating in the lower troposphere and cooling in the middle and upper troposphere. The forecasts represent these features reasonably well. As noticed in other heat budget parameters, the forecasts produce comparatively weak heating and cooling patterns. Nevertheless, a significant feature noticed in this study needs considerable attention. The diabatic heating depicts anomalous cooling in the lower levels $(800-600 \mathrm{hPa})$, which impedes the vertical exchange of heat flux transfer from the surface to upper layers of the atmosphere. This influences the monsoon circulation considerably. Earlier studies revealed that the vertical variation of diabatic heating distribution shows a boundary layer maximum in the tropical latitudes. This heating is accounted by the turbulent heat transfer from the relatively warm ocean and land surfaces. Apart from mid-tropospheric maximum another cooling maximum around $200 \mathrm{hPa}$ level is also noticed in the monsoon region, which is due to radiational effects produced by the clouds.

All forecasts slightly underpredict the budget parameters. The significant aspect persistent in the present study is the anomalous diabatic cooling depicted by the model over the monsoon region. Earlier studies related to the Indian summer monsoon (Mohanty et al 1983; Rao 2001) indicate diabatic heating in the lower troposphere of the monsoon region during its active phase. As explained earlier, the diabatic heating in the lower troposphere is due to the turbulent exchange of sensible heat flux between surface and upper layers. The diabatic cooling in the lower troposphere hinders the vertical transfer of sensible heat. This is due to the use of zonal mean climatological clouds in the model, which produce abundant radiational 
MOISTURE BUDGET

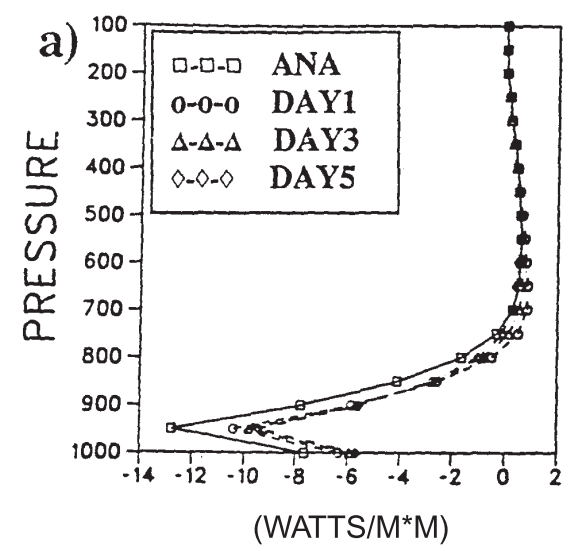

Figure 6. As in figure 5, but for moisture budget terms source/sink.

cooling. This appears to stabilize the model's midtroposphere and is also partly responsible for the cold bias near $700 \mathrm{hPa}$ level. However, experiments performed at NCEP (White 1988) divulge the improvement in the lower and middle troposphere cooling by introducing the interactive clouds. The diabatic heating in the upper levels due to release of latent heat of condensation is also not represented properly in the model.

The vertical profile of moisture budget parameters for the monsoon season of 1995 is illustrated in figure 6 . The horizontal flux convergence of moisture indicates maximum in the boundary layer, minimum around $700 \mathrm{hPa}$ and thereafter it depicts weak flux divergence up to $300 \mathrm{hPa}$. Despite underpredicting the moisture flux convergence, the forecasts represent the characteristic features of horizontal flux divergence of the moisture realistically. The large influx of moisture to the monsoon region leads to excessive condensation, which in turn enhances diabatic heating and eventually maintains the monsoon circulation. The moisture source/sink (or diabatic heating due to moisture) is represented in figure $6(\mathrm{~b})$. It is noticed that the diabatic heating due to moisture depicts cooling in the lower and upper troposphere except between $900-700 \mathrm{hPa}$ layer where heating is dominant. The model forecasts simulate the diabatic heating pattern fairly well. However, the forecasts slightly underpredict it.

The interesting finding of the study is the anomalous pattern delineated by diabatic heating. Earlier studies (Mohanty et al 1983; Rao 2001) revealed that the monsoon region is dominated by diabatic heating. However, the analysis and forecasts show cooling in the planetary boundary layer and the upper troposphere. Due to condensation of moisture, latent heat is released in the upper tro-
JJA 1995

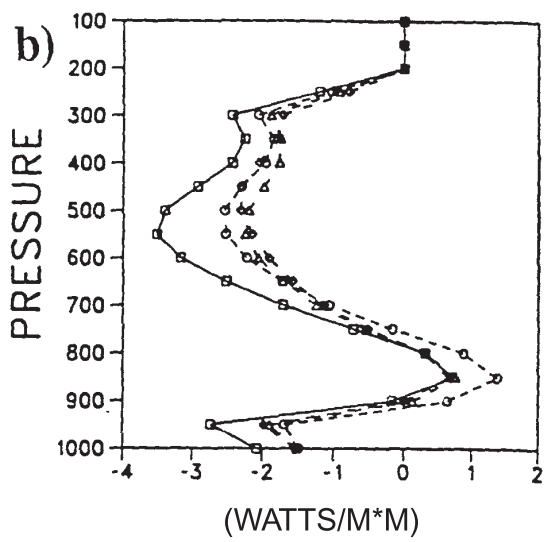

(a) Horizontal flux divergence of moisture, (b) moisture posphere, which heats up the atmosphere. Nevertheless, the present study shows excess evaporation compared to condensation which in turn results to cooling. Since the analysis also reflects the same feature, this appears to be due to deficiencies of the model. The shallow convection, which is expected to enhance the condensation mechanism, appears to be ineffective. The impact of the diabatic cooling in the lower and middle troposphere would be inhibition of the exchange of moisture between the lower and upper levels. As a result, the model produces weak moisture convergence over the monsoon season. The monsoon circulation to a large extent is thus controlled and maintained by two significant parameters, namely the diabatic heating and the moisture flux convergence. Since these two parameters are underestimated, their effect is noticed in the circulation. Thus, the model produces weak monsoon circulation. The moisture flux convergence precedes the establishment of diabatic heating source over the monsoon regime (Mohanty et al 1983; Pearce and Mohanty 1984). It appears that the moisture convergence plays a dominant role in determining the diabatic heating pattern, which in turn maintains the monsoon circulation. The vertical profiles of various parameters in the heat and moisture budgets are in agreement with earlier studies (Savijarvi 1980, 1981), despite the fact that we can not have a one-to-one correspondence with the studies of Savijarvi $(1980,1981)$ as they are pertaining to Europe, North America and Atlantic using ECMWF data sets. However, our comparison with a recent study (Rao 2001) evinces that these profiles are in good agreement with the monsoon climatology, except for the anomalous diabatic cooling zone in the lower middle troposphere delineated by the NCMRWF model which needs some correction. 

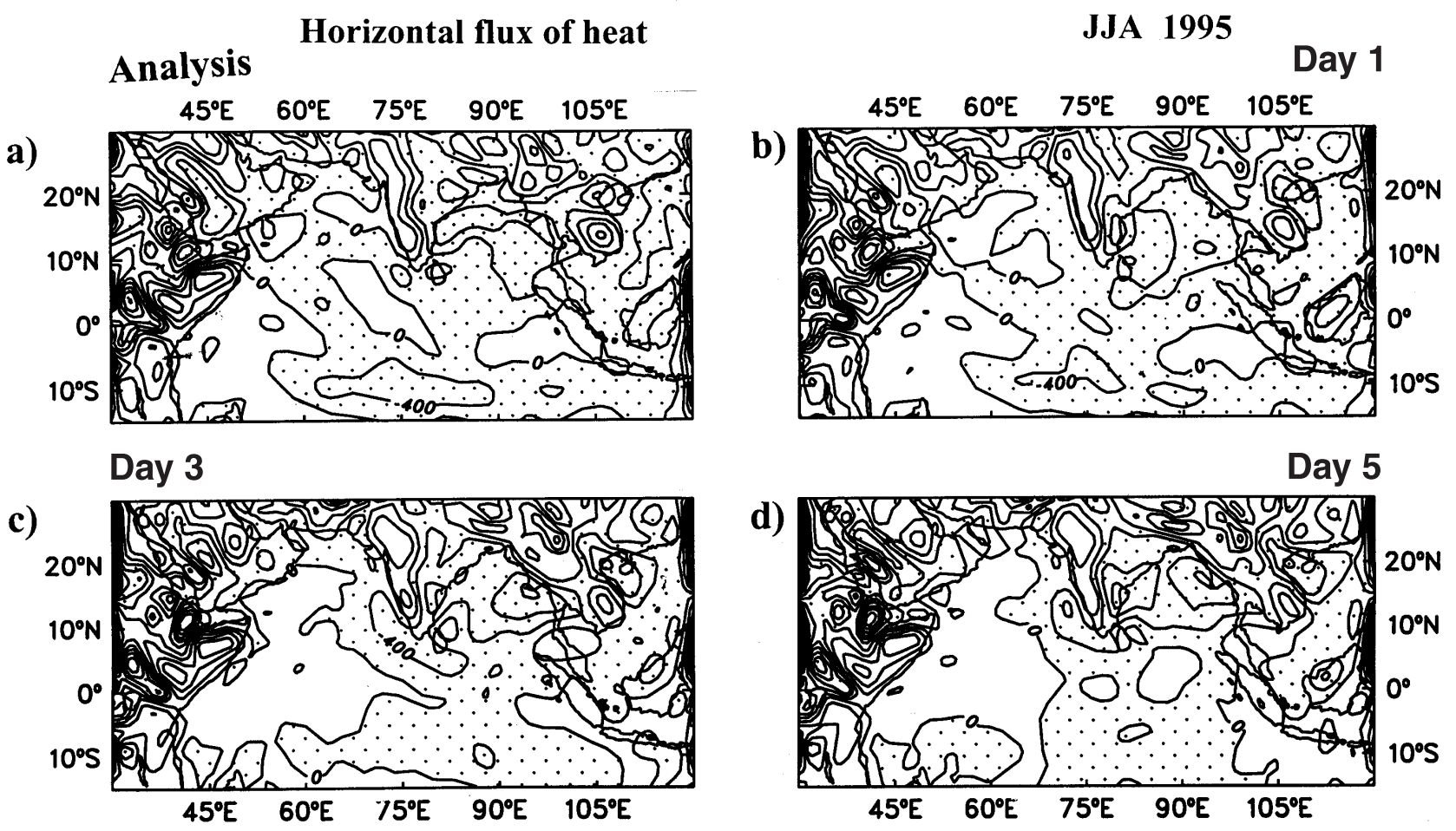

Figure 7. Geographical distribution of vertically integrated horizontal flux divergence of heat for JJA 1995 (units: Watt $\mathrm{m}^{-2}$ ); (a) Analysis, (b) Day 1 forecast, (c) Day 3 forecast, (d) Day 5 forecast. (Contour interval: 400, negative values are shaded).

The performance of the NCMRWF model is further analyzed through vertically integrated (1000$100 \mathrm{hPa}$ ) geographical distributions of heat and moisture budget parameters for JJA 1995 and 1993. The horizontal flux divergence of heat for JJA 1995 (figure 7) shows that the entire monsoon domain is characterized by convergence of heat barring east Africa where predominant divergence is noticed. The convergence maxima of heat are noticed over the Bay of Bengal and Arabian Sea. The model forecasts also evince these features. In agreement with the systematic errors, the flux convergence of heat is underestimated in the day 1 and day 3 forecasts compared to the analysis. The lower tropospheric cooling and weak transequatorial flow produced by the model are responsible for this underestimation. Incidentally, the horizontal flux divergence of heat during JJA 1993 (figure 8) also indicates similar features. The notable difference between 1995 and 1993 is that the flux divergence maximum over the Bay of Bengal is stronger during 1993.

The geographical distribution of diabatic heating for JJA 1995 is depicted in figure 9. The monsoon domain is characterized by diabatic heating during the active phase of the summer monsoon. The strong moisture influx coupled with intense rising motion and release of latent heat are the essential factors in sustaining the diabatic heat sources over the monsoon region. The diabatic heating denotes two maximum over the Arabian Sea and Bay of Bengal. Cooling is noticed over the southern Indian Ocean and off east Africa. It is very interesting to notice that the strong flux convergence zones of heat are identified with intense diabatic heating. In agreement with the error pattern, the diabatic heating is underestimated in the day 1 forecast. However, the model retains the analyzed heating by day 5 . The error characteristics are identical during 1995 and 1993. The diabatic heating over the Bay of Bengal is more intense during 1993 (figure 10). This is attributed to the strong horizontal flux of heat noticed during the same period over the Bay of Bengal.

The horizontal flux divergence of moisture for JJA 1995 (figure 11) indicates that most of the monsoon domain is characterized by convergence of moisture with maxima over the Bay of Bengal and Arabian Sea. However, flux divergence is noticed off east Africa. The differential heating between the south Asian continent and the adjoining tropical Indian Ocean necessitates the transequatorial flow off east Africa. This flow is primarily responsible for the transport of mass and moisture over the monsoon domain from the Southern Hemisphere. The model forecasts represent these features reasonably well. However, in tandem with the systematic errors in the moisture and wind fields, the 
Horizontal flux of heat

JJA 1993

Analysis

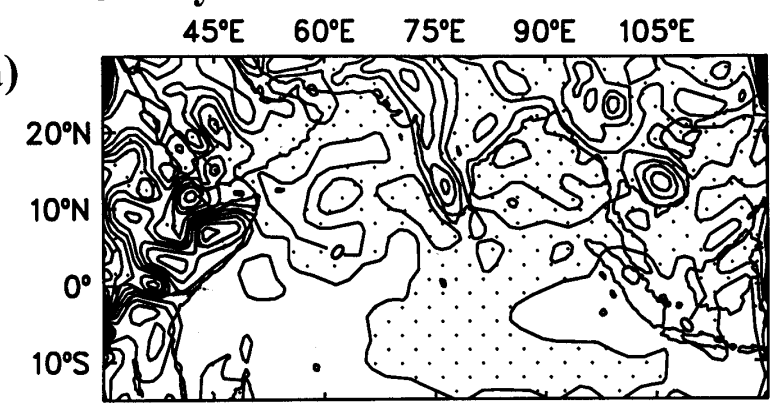

b)

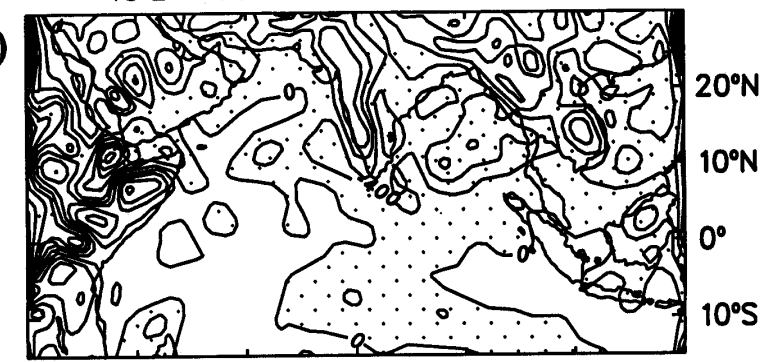

\section{Day 3}

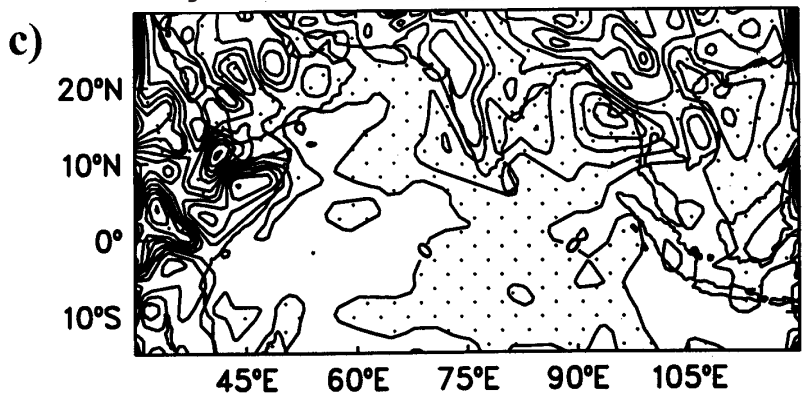

d)

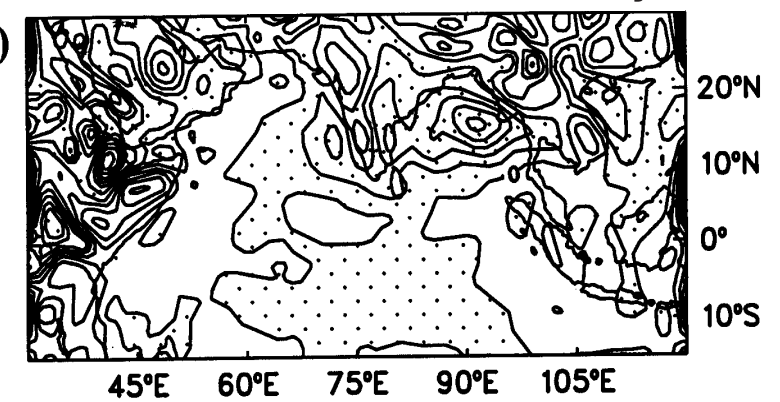

Figure 8. As in figure 7, but for JJA 1993.

Analysis

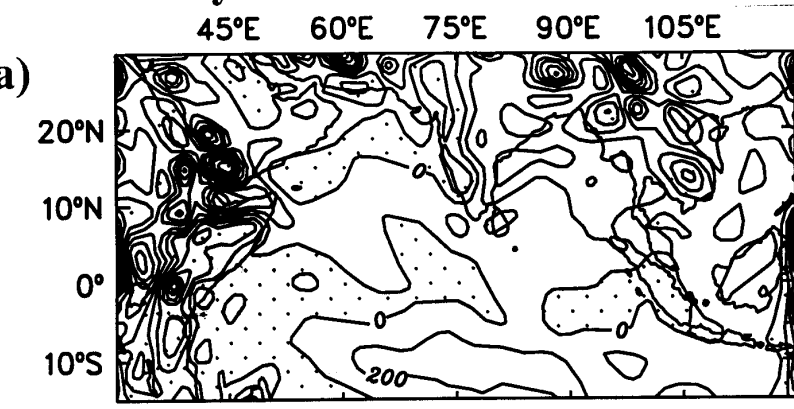

\section{Day 3}

c)

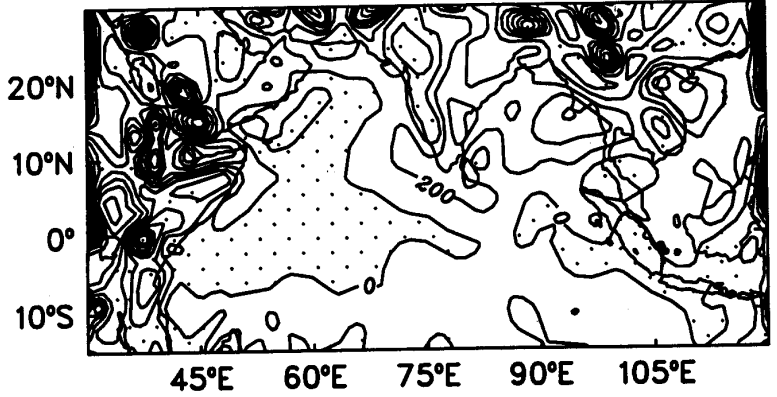

JJA 1995

$45^{\circ} \mathrm{E} \quad 60^{\circ} \mathrm{E} \quad 75^{\circ} \mathrm{E} \quad 90^{\circ} \mathrm{E} \quad 105^{\circ} \mathrm{E}$

b)

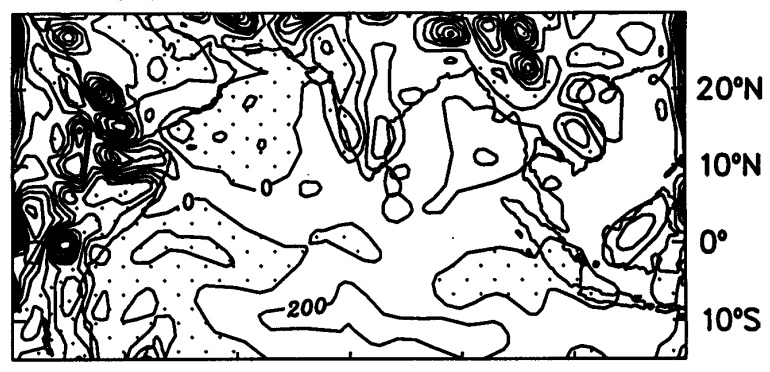

Day 5

d)

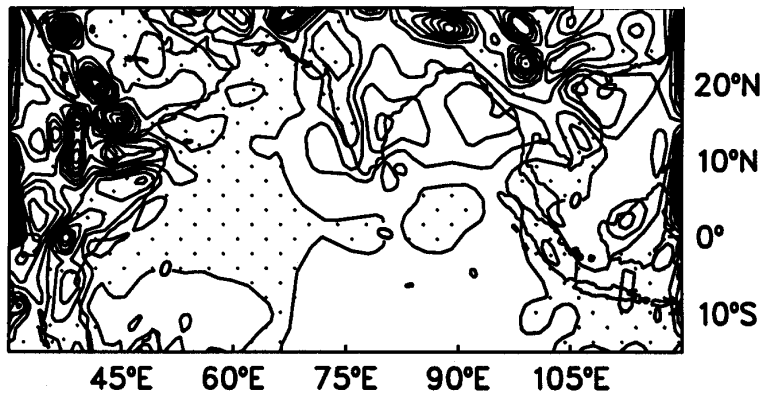

Figure 9. Geographical distribution of vertically integrated diabatic heating for JJA 1995 (units: Watt m $^{-2}$ ); (a) Analysis, (b) Day 1 forecast, (c) Day 3 forecast, (d) Day 5 forecast. (Contour interval: 200, negative values are shaded). 


\section{Analysis}

Diabatic heating

JJA 1993

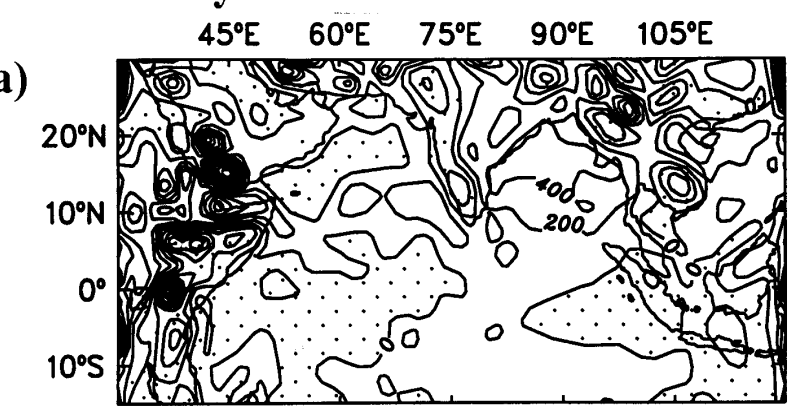

Day 3

c)

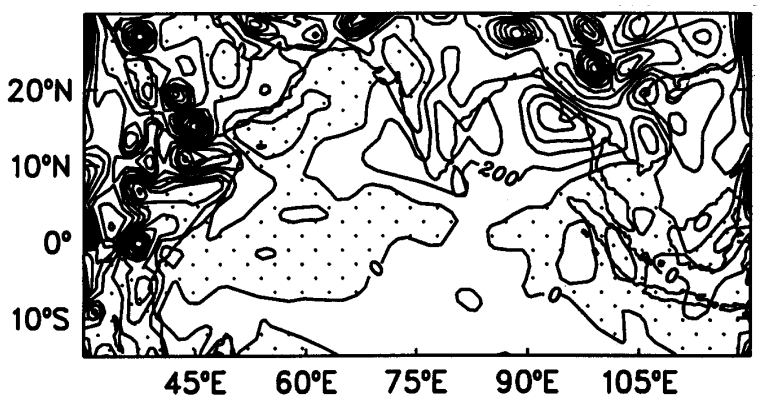

b)

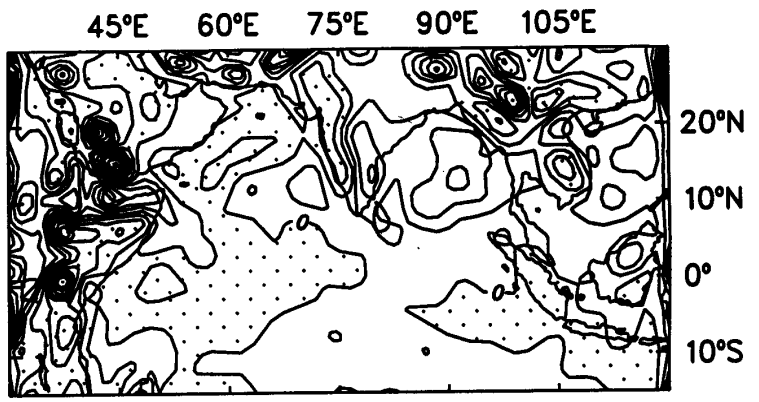

Day 5

d)

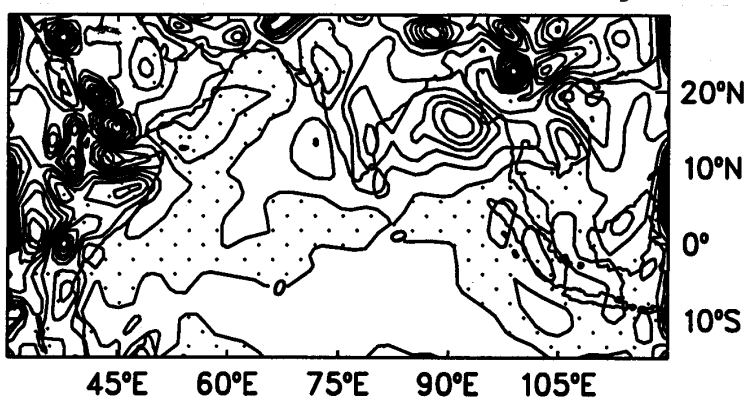

Figure 10. As in figure 9, but for JJA 1993.

Analysis

Horizontal flux of moisture

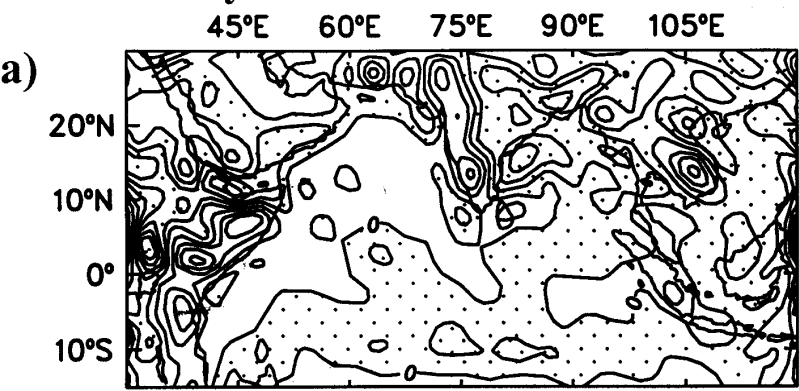

Day 3

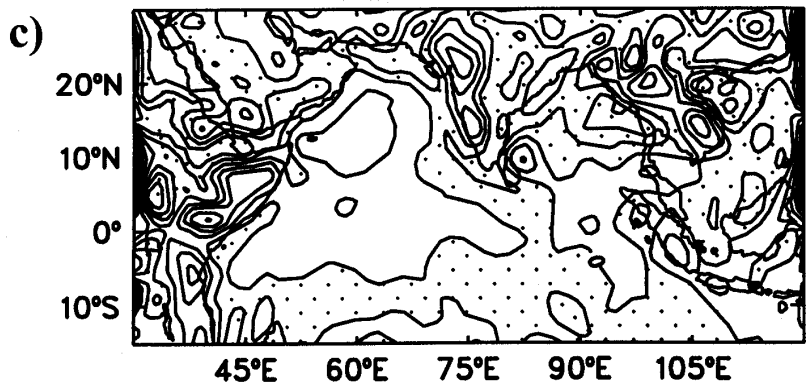

JJA 1995

Day 1

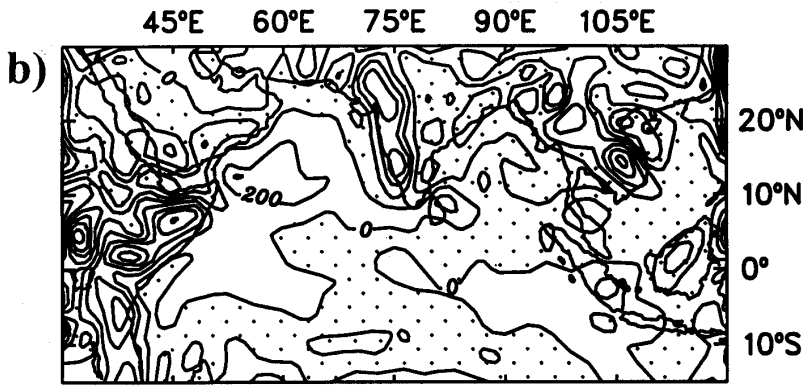

Day 5

d)

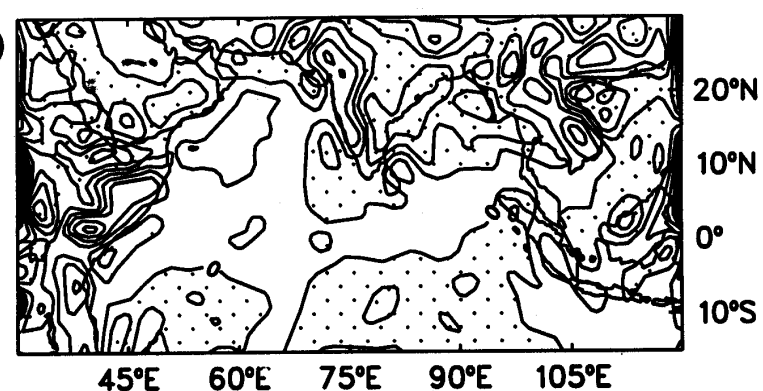

Figure 11. Geographical distribution of vertically integrated horizontal flux divergence of moisture for JJA 1995 (units: Watt $\mathrm{m}^{-2}$ ); (a) Analysis, (b) Day 1 forecast, (c) Day 3 forecast, (d) Day 5 forecast. (Contour interval: 200, negative values are shaded). 
Horizontal flux of moisture

Analysis

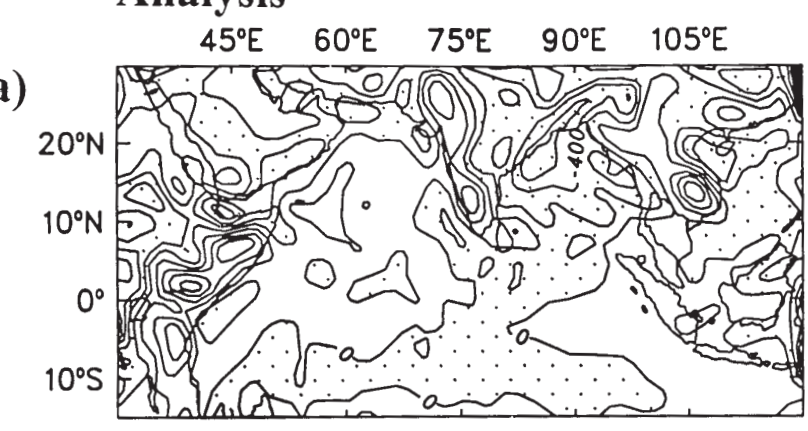

Day 3

c)

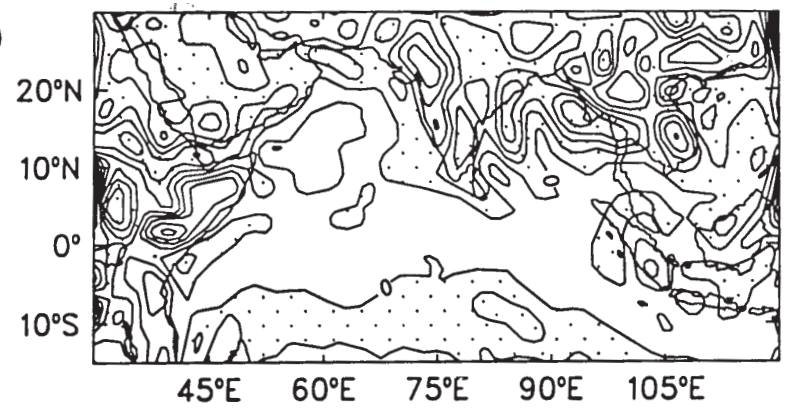

JJA 1993

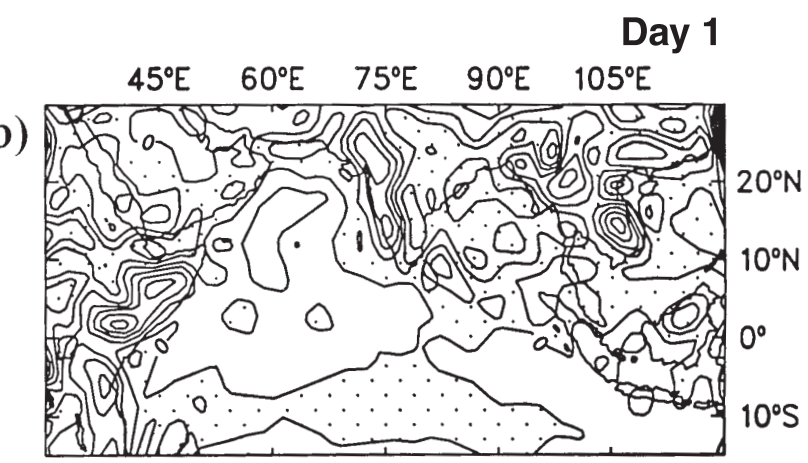

Day 5

d)

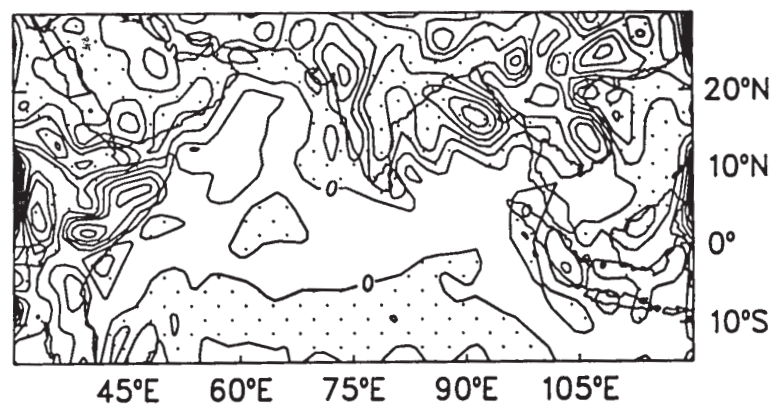

Figure 12. As in figure 11, but for JJA 1993.

day 1 and day 3 forecasts underestimate the flux convergence. This underestimation in the moisture flux is accounted by the general drying of the model atmosphere, apart from the feeble cross-equatorial flow. Interestingly, the flux divergence of moisture during JJA 1993 (figure 12) also indicates similar features. However, as noticed in the case of the heat budget terms, the Bay of Bengal maximum is pretty intense during 1993.

The volume integrated $\left(15^{\circ} \mathrm{S}-45^{\circ} \mathrm{N}, 30^{\circ} \mathrm{E}-120^{\circ} \mathrm{E}\right.$, $1000-100 \mathrm{hPa}$ ) heat budget parameters for JJA 1995 are depicted in figure 13. It could be seen from the volume integrated domain of heat budget, that the horizontal flux divergence and the adiabatic conversion of APE to KE contribute significantly to the heat budget. Since the temperature forecasts depict a cold bias in the lower and middle troposphere over the monsoon region with increase in forecast length, the model is underestimating the heat budget parameters with increase in the forecast period. Consequently, the corresponding changes in the circulation features are also noticed. As enunciated in the heat and moisture budgets the diabatic heating and the moisture convergence together sustain the monsoon circulation. In general, the forecasts underpredict the heat budget parameters with increase in the forecast period. The horizontal flux of heat, the adiabatic conversion of kinetic energy from APE and the diabatic heating delineate the characteristic systematic errors of the model. By day 5 there appears to be marginal retention of the strength, but yet comparatively weaker, than that of the analysis.

The volume integrated moisture budget parameters for JJA 1995 are illustrated in figure 14 . The forecasts reveal the underestimation of horizontal flux divergence of moisture with the increase in the forecast period, which is due to the general drying of the model atmosphere with increase in the forecast period. This is evident in the volume integrated flux divergence of the moisture. In order to sustain the monsoon circulation, persistent moisture convergence and intense diabatic heating are indispensable features. The underprediction of these features indicates that the summer monsoon circulation is enfeebled in various ranges of forecasts.

Figure 15 delineates the observed (Xie and Arkin 1996) total seasonal (JJAS) rainfall over the Indian summer monsoon domain for 1995 and 1993. The maximum rainfall is noticed over the northern Bay of Bengal, northeast India and adjoining Myanmar region. This is in agreement with the monsoon climatology. The rainfall pattern is almost similar in both years except over the Bay of Bengal and eastern Indian Ocean where excess rainfall is noticed. This is in confirmation with the patterns of vari- 

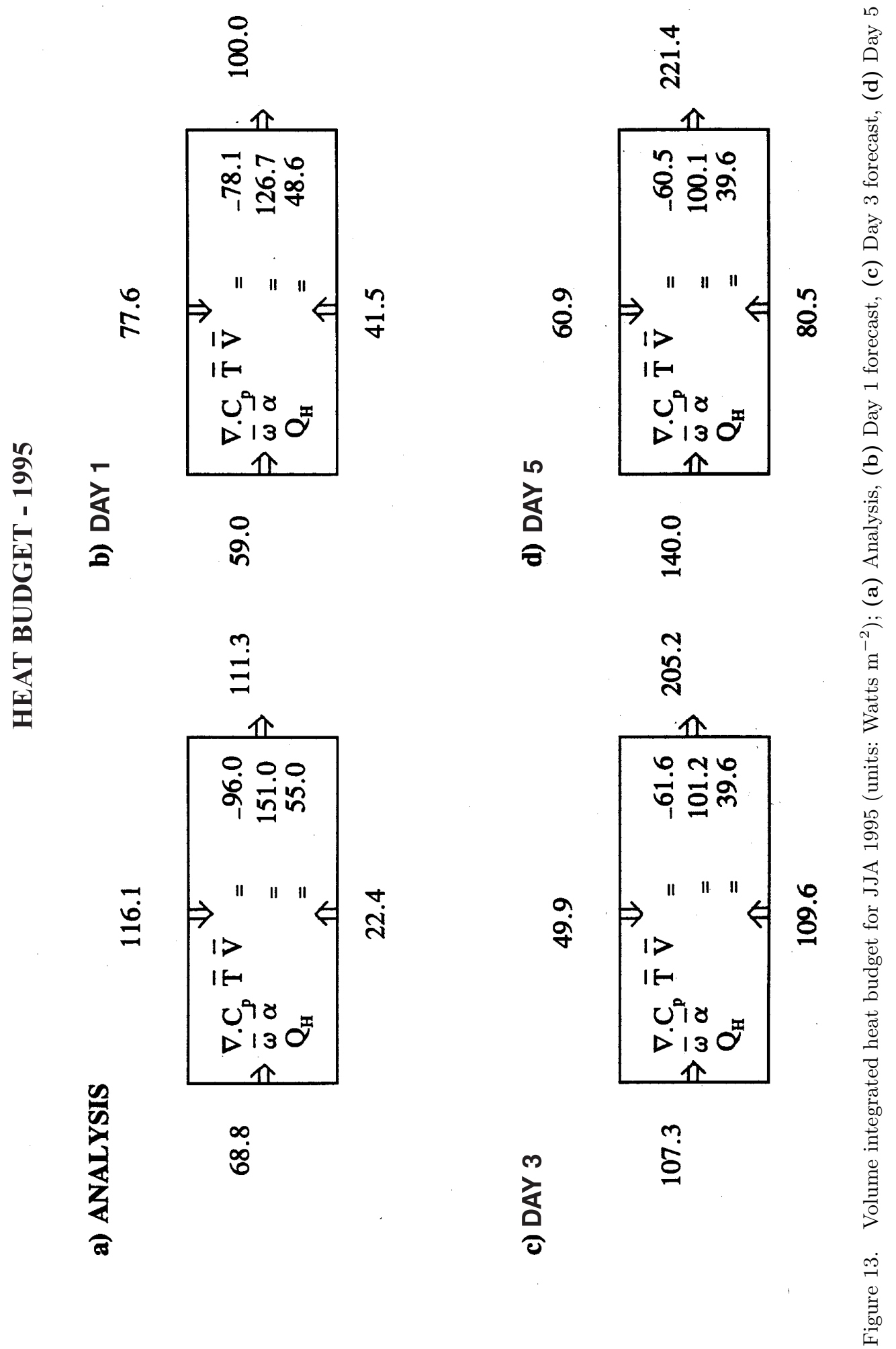


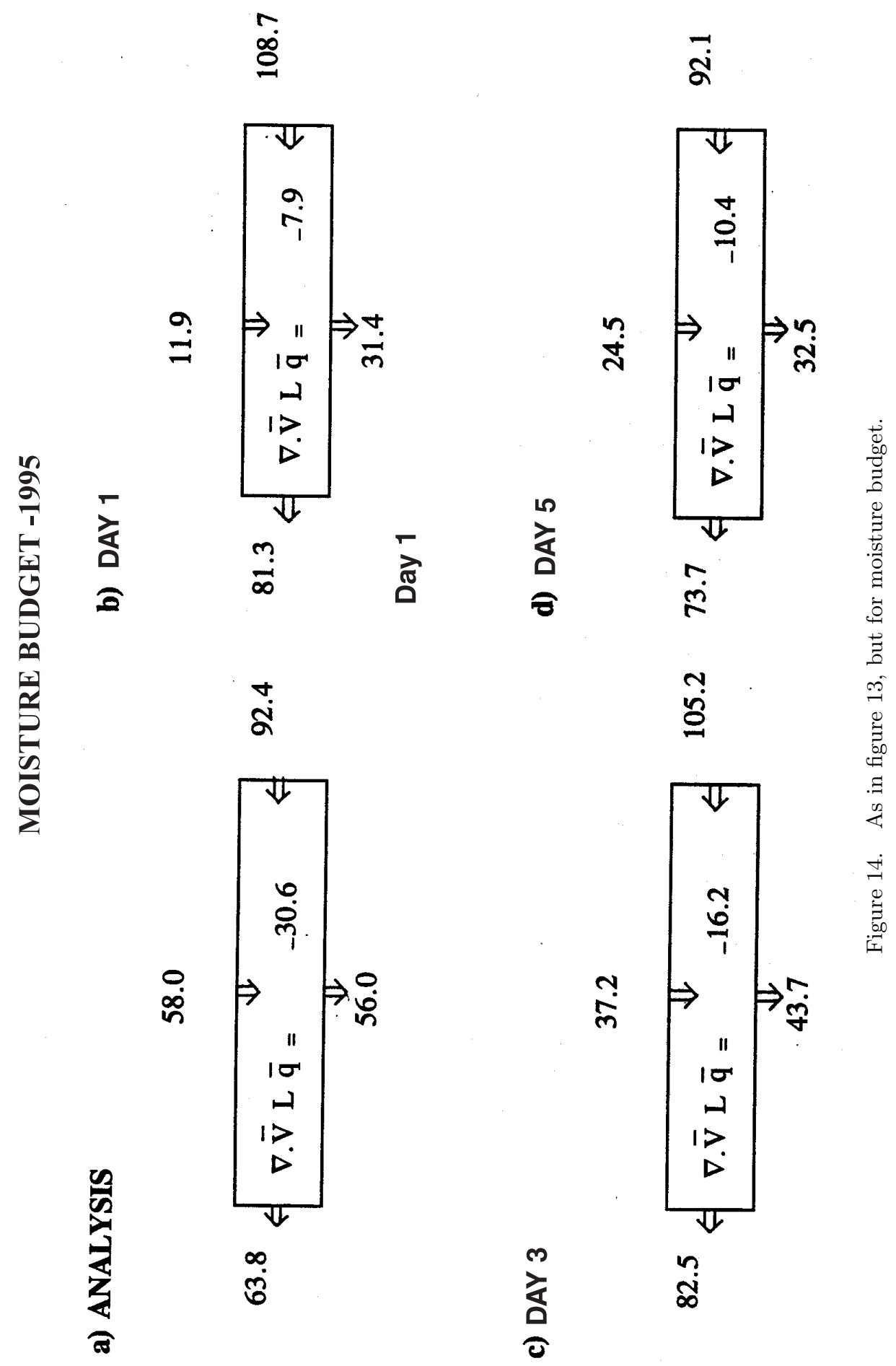


Xie-Arkin rainfall (JJAS)
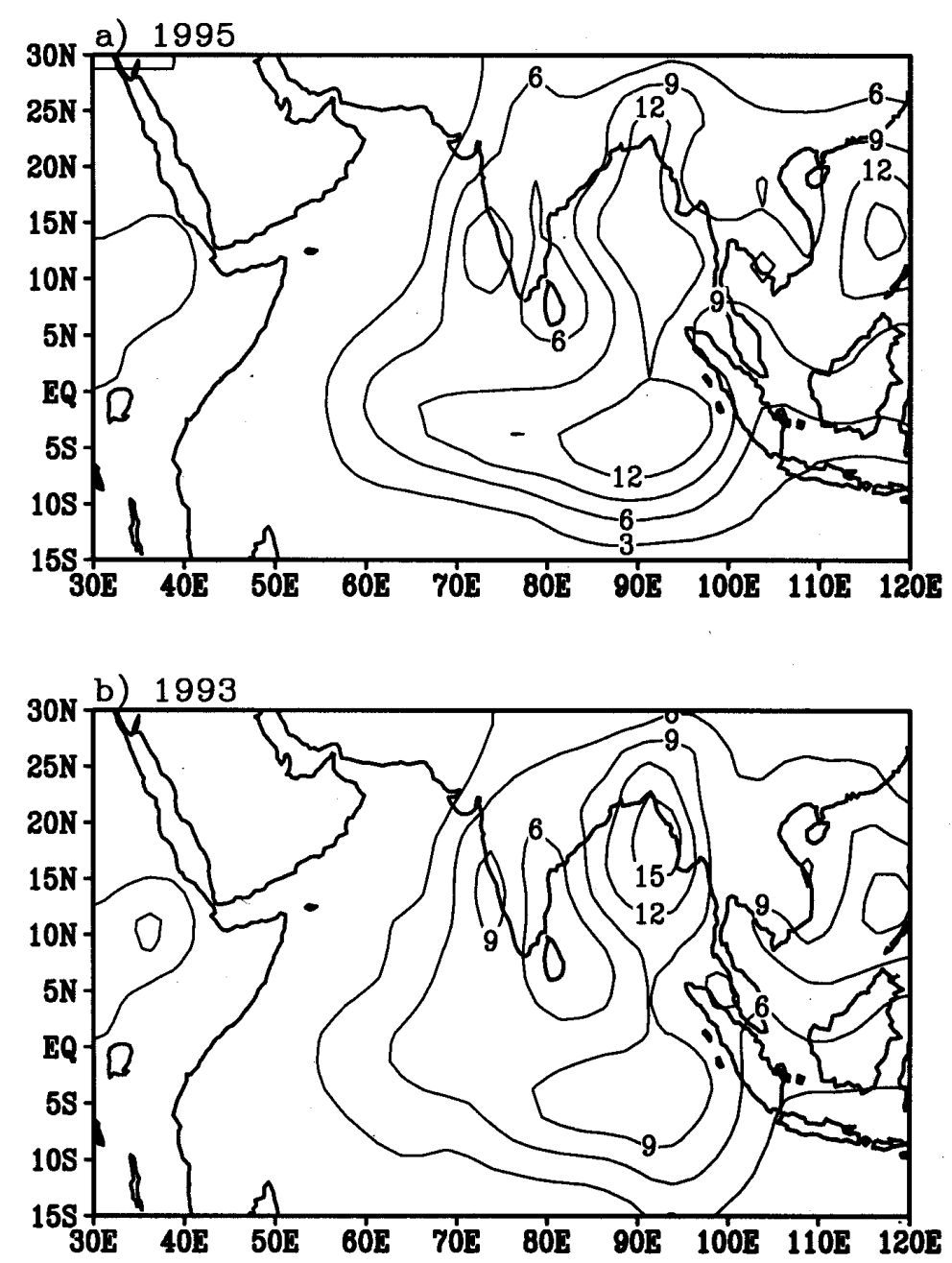

Figure 15. Xie-Arkin total seasonal (JJAS) observed rainfall (units: mm); (a) 1995, (b) 1993. (Contour interval: 3).

ous parameters of large-scale balances of heat and moisture.

\section{Conclusions}

The study elucidates the following aspects. Broadly, it addresses the important aspects of the NCMRWF model namely, the representation of seasonal mean monsoon circulation, the influence of systematic errors on this circulation, and the interseasonal variability of the summer monsoon.

The seasonal mean large scale balances of heat and moisture are in agreement with the summer monsoon climatology. Further, the balance requirements are fairly met at various forecast lengths. The study adduces that the summer monsoon domain is characterized as the sink of heat and moisture. The forecasts corroborate this aspect.

The systematic errors of the temperature forecasts depict cooling in the entire troposphere barring the upper troposphere above $200 \mathrm{hPa}$ level. The moisture forecasts indicate general drying of the model atmosphere in the lower troposphere over the monsoon domain. The effect of these errors is weakening of westerlies and narrowing the zone of easterlies over the monsoon. In order to sustain the monsoon circulation, the moisture flux convergence, the heat flux convergence and the diabatic heating are the crucial parameters. Since the model is underestimating these parameters, the monsoon circulation produced by the model gets feeble with the increase in the forecast period. Furthermore, the diabatic heating depicted by the heat and moisture budgets show anomalous cooling pattern over 
the monsoon region. Earlier studies reveal that the monsoon region is characterized by diabatic heating in the lower as well as upper tropospheres. The present study significantly deviates from them in the representation of diabatic heating. Since the analysis also delineates the same tendency, the presence of diabatic cooling is noticed even in the first guess. This is due to deficiencies in the representation of long wave radiation and shallow convection in the model. This entails the improvement in the parameterization of these two schemes in order to represent the monsoon circulation realistically.

Apart from the deficiencies of the model the study also elucidates the positive aspects. It is also demonstrated that the model can capture the interseasonal variability of the summer monsoon between a couple of normal monsoon seasons. As noticed, the monsoons of 1995 and 1993 are normal with slightly excess rainfall over the Bay of Bengal during 1993. The net tropospheric budget terms in the large-scale balances of heat and moisture under consideration evinced this aspect lucidly. The variability depicted by the diagnostic fields is in tandem with the observed variability in terms of rainfall. This is an interesting aspect of the model.

\section{Acknowledgements}

The authors express their profound sense of gratitude to the NCMRWF for providing computing and data facilities to carry out the present study. This work is partially financed by the Department of Science and Technology (DST), India. The authors are also grateful to the anonymous reviewers for their helpful suggestions, which improved the clarity of the paper.

\section{References}

Kanamitsu M and Krishnamurti T N 1978 Northern summer tropical circulations during drought and normal rainfall months; Mon. Weather Rev. 10 331-347

Krishnamurti T N, Bedi H S and Subramaniam M 1989 The summer monsoon of 1987; J. Climate 2(4) 321-340
Krishnamurti T N, Bedi H S and Subramaniam M 1990 The summer monsoon of 1988; Meteorol. Atmos. Phys. 42 1937

Mohanty U C, Dube S K and Singh M P 1983 A study of heat and moisture budgets over the Arabian Sea and their role in the onset and maintenance of summer monsoon; J. Meteorol. Soc. Japan 61 208-211

Mohanty U C and co-authors 1995 Medium range prediction of atmosphere system over Indian region by NCMRWF system, Proceedings of Fifth regional workshop on Asian/African monsoon emphasizing training aspects (WMO Tropical Meteorology Research Programme Series Report no:52, WMO/TD-NO: 698), 51-62

Nitta T 1970 A study of generation and conversion of eddy available potential energy in the tropics; J. Meteor. Soc. Japan $486524-528$

O'Brien J J 1970 Alternative solutions to classical vertical velocity problem; J. Appl. Met 9 197-203

Pearce R P and Mohanty U C 1984 Onsets of the Asian summer monsoon 1979-82; J. Atmos. Sci. 419 1622-1639

Ramesh K J, Mohanty U C and Rao P L S 1996 A study on the distinct features of the Asian summer monsoon during the years of extreme monsoon activity over India; Meteorol. Atmos. Phys. 59 3-4 173-183

Rao K V and Rajamani S 1972 Study of heat sources and sinks and the generation of available potential energy in the Indian region during the southwest monsoon season; Mon. Weather. Rev. 100 383-388

Rao P L S, Mohanty U C and Ramesh K J 1998 Mean dynamical characteristics of the Asian summer monsoon with a global analysis-forecast system; Meteor. Atmos. Phys. 68 1-2 57-77

Rao P L S 2001 The energetics of Asian summer monsoon; PAGEOPH 158 5-6 965-988

Savijarvi H 1980 Energy budget calculations and diabatic effects for limited areas computed from ECMWF analyses and forecasts; Proceedings of the workshop: Diagnostics of diabatic processes ECMWF, 115-134

Savijarvi H 1981 The energy budgets in North America, North Atlantic and Europe based on ECMWF analyses and forecasts, ECMWF Tech Report No: 27

Slingo J M, Mohanty U C, Tiedtke M and Pearce R P 1988 Prediction of the 1979 summer monsoon onset with modified parameterization schemes; Mon. Weather. Rev. 116 328-346

Tiedtke M, Heckley W A and Slingo J M 1988 Tropical forecasting at ECWMF: The influence of physical parameterization on the mean structure of forecasts and analyses; Q. J. R. Meteorol. Soc. 114 639-664

White G H 1988 On the performance of the NMC medium range forecast model in midlatitudes. Palmen memorial symposium on extratropical cyclones, Helsinki, Finland; American Meteorological Society 305-308

Xie P and Arkin P 1996 Analysis of global monthly precipitation using gauge observations, satellite estimates, and numerical model predictions; J. Climate $9840-$ 858 